\title{
Performance Analysis of A Modified OFDM System with Cross Polarization Diversity Structure
}

\author{
Deock-Ho Ha ${ }^{1}$, Kyu-il Han ${ }^{2}$
}

\begin{abstract}
In this paper, we proposes a modified OFDM, CPD-OFDM (Orthogonal Frequency Division Multiplexing with cross polarization diversity structure), system combating the system performance degradation due to the frequency offset. The cross polarization diversity structure composed with 2-pairs cross polarized circular antenna in each transceiver has the characteristic that it can remarkably remove the odd time reflected waves in each receiving end. The cross circular polarization diversity structure can reduce the time delay spread and ICI. Therefore, the proposed CPD-OFDM system can improve the system performance as well as the spectrum efficiency. In order to investigate the performance improvement of OFDM system due to the frequency offset, computer simulation and theoretical analysis were conducted. From this analysis, it can be seen that the CPD-OFDM system performance has been improved by $1 \sim 3[\mathrm{~dB}]$ compared to that of the conventional OFDM system.
\end{abstract}

Keywords - frequency offset, removing delayed waves, cross polarization diversity structure, system performance improvement

\section{Introduction}

Studies on high quality digital transmission system is still eagerly conducted to realize the broadband multimedia communication system with more high speed. The OFDM is a special case in multicarrier transmission, where a single data stream is divided into many subcarriers and then transferred in a parallel way. Therefore, the OFDM symbol has the long interval between symbols and this reduces the interference between adjacent symbols [1]. And also, the OFDM system reduces the necessary bandwidth by using the orthogonality between the sub-channels [2]. For this orthogonality between the sub-channels, the OFDM uses cyclic prefix as a guard interval. Usually, guard interval is chosen as larger scale than the channel delay spread value, so that multipath components occurred from a symbol do not interfere with the next adjacent symbol.

${ }^{1}$ Deock-Ho Ha/ Dept. of Information and Communications Engr.

College of Engineering/PuKyoung National University

Korea, Republic of

${ }^{2}$ Kyu-il Han/Dept. of Mechanical System Engr,

College of Engineering/PuKyoung National University

Korea, Republic of
In this guard interval, a copy of the cyclic extension of the frame is fitted. This cyclic prefix can keep the orthogonality between the sub-channels [3]. In case of the cyclic prefix is set larger than the time delay of channel, it keeps the orthogonality between sub-channels [4]. However, in case of the multipath delay becomes larger than the guard interval, the phase transitions of the delayed path fall within the fast Fourier transform (FFT) interval of the receiver. Thus, it makes channel interference and then caused system performance degradation [5].

In this paper, we proposed a modified OFDM compose of cross polarization diversity structure (CPD-OFDM) for reducing the frequency offset occurred in conventional OFDM system. The proposed system can minimize the delay spread and the interference of reflected waves as well as ICI in conventional OFDM system. From the analysis results of system performance, it was found that the proposed CPDOFDM system shows better performance compared to the conventional OFDM system.

\section{Conventional OFDM System}

In OFDM system, the $k^{\text {th }}$ subcarrier $f_{k}$ can be represented as follows:

$$
f_{k}=\frac{k}{N T_{d s}}=\frac{k}{T_{s}}
$$

Where, $N$ is the number of subcarriers, $T_{d s}$ is the data symbol duration and $T_{s}$ is the OFDM symbol duration. Therefore, the baseband signal of the OFDM $s(t)$ can be represented as follows [6]:

$$
s(t)=\sum_{n=-\infty}^{\infty} \sum_{k=0}^{N-1} \frac{C}{\sqrt{T_{s}}} d_{n, k} e^{j 2 \pi f_{k} t} p\left(t-n T_{s}\right)
$$

In equation (3), $\mathrm{C}$ is the constant related to the power of the signal, and $d_{n, k}$ is the data symbol allocated to the $k^{\text {th }}$ subcarrier in the $n^{\text {th }}$ symbol duration $\left[n T_{s},(n+1) T_{s}\right]$. The $p(t)$ represents pulse shaping function. An OFDM frame signal $s_{i}(t)$ in the period of $\left[n T_{s},(n+1) T_{s}\right]$ can be represented as follows:

$$
s_{i}(t)=\sum_{k=0}^{N-1} \frac{C}{\sqrt{T_{s}}} d_{n, k} e^{j 2 \pi f_{k} t}
$$


where, $n T_{s} \leq t(n+1) T_{s}$. Therefore, the sampled signal of the OFDM frame signal $s(m)$ can be represented as follows:

$$
s(m)=\sum_{k=0}^{N-1} \frac{C}{\sqrt{T_{s}}} d_{n, k} e^{j 2 \pi k m / N}
$$

where, $f_{k}=\frac{k}{N T_{d s}}, m=0,1, \ldots, N-1$.

This equation (4) has the same results that can be obtained from inverse discrete Fourier transform (IDFT) of data symbol $\mathrm{d}_{\mathrm{n}, \mathrm{k}}$. It means that the modulation of the subcarrier can be achieved by the IDFT of the data symbol. All data symbols in the subcarriers can be transmitted simultaneously using the orthogonality between subcarriers if there is no distortion in the channel. In order to eliminate ICI, the guard interval should be inserted in each OFDM frame. This guard interval should be longer than the time delay spread of channel [5]. Usually, the system performance OFDM cannot be improved even though the SNR (signal to noise ratio) increase higher when the delay spread is longer than the guard interval. The orthogonality of subcarriers cannot be kept and there happens ICI when the time delay is longer than the guard interval.

\section{CROSS Polarization Diversity}

\section{A. Basic Concept}

The proposed cross polarization diversity structure is composed of other phase difference using vertical polarization and horizon polarization. It also divides the sub-channels into a right-handed circular polarization (RHCP) channel and a left-handed circular polarization (LHCP) channel that alternate each other. Vertical polarization phase is almost not change when propagation reflects off Brewster angle but horizon polarization makes 180 degree phase inversion occur. Because of the circular polarization antenna is composed with 90 degree transition of the vertical and horizontal antenna, RHCP change to LHCP when it is odd-time reflection, vice versa [7], [8]. It is also well known that the polarization diversity can reduce the interference of reflected waves and minimizes the multipath fading in indoor/outdoor wireless channel [9].

\section{B. CPD-OFDM System [10]}

The spectrums of the sub-channels are overlapped by a spectrum of adjacent sub-channels in the conventional OFDM system. This means that a small amount of frequency offset can cause the degradation of the system performance because the orthogonality between the sub-channels could not be kept. If the overlapping of a sub-channel spectrum can be reduced, then we can minimize the degradation due to the frequency offset. For this reason, the modified OFDM system using cross polarization diversity structure is proposed. Figure 1 shows the structure of the CPD-OFDM and Figure 2 shows a subchannel spectrum of the proposed system.
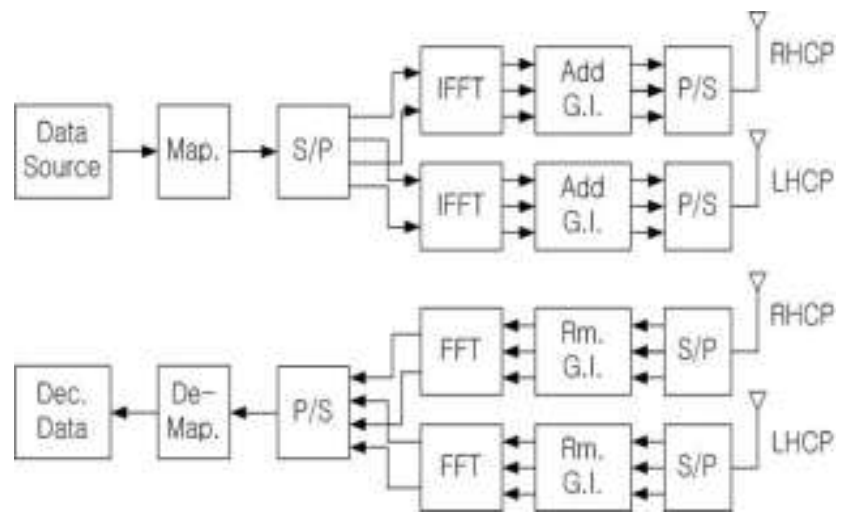

Figure 1. Structure of the CPD-OFDM system

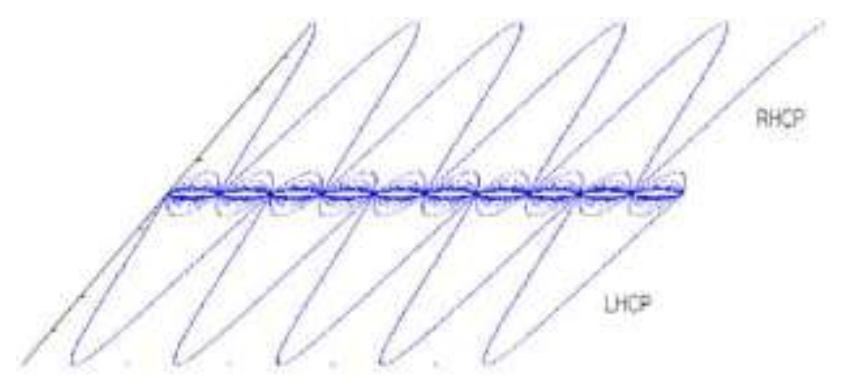

Figure 2. Spectrum of subcarrier in CPD-OFDM system

The proposed CPD-OFDM system divides the sub-channels into a RHCP channel and a LHCP channel that alternate each other. The signals of the RHCP channel $s_{R}(t)$ and the signal of the LHCP channel $S_{L}(t)$ can be achieved from the equation (2) as follows:

$$
\begin{aligned}
& s_{R}(t)=\sum_{n=-\infty}^{\infty} \sum_{k=0}^{(N-1) / 2} \frac{C}{\sqrt{T_{s}}} d_{n,(2 k)} e^{j 2 \pi f(2 k) t} p\left(t-n T_{s}\right) \\
& s_{L}(t)=\sum_{n=-\infty}^{\infty} \sum_{k=0}^{(N-1) / 2} \frac{C}{\sqrt{T_{s}}} d_{n,(2 k+1)} e^{j 2 \pi f_{(2 k+1)} t} p\left(t-n T_{s}\right)
\end{aligned}
$$

Figure 3 shows the model of the channel in the CPD-OFDM system. In this figure $h_{i j}$ is the transfer function that has the characteristics of transmission in the $i^{\text {th }}$ channel and reception by the $\mathrm{j}^{\text {th }}$ channel.

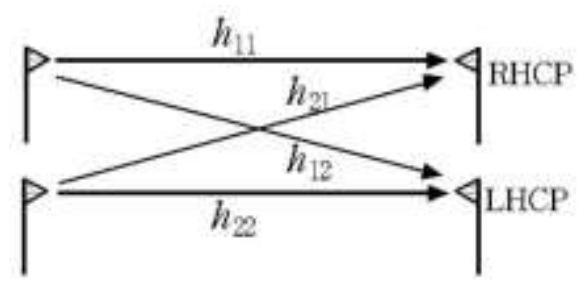

Figure 3. Channel model of the CPD-OFDM system 
Therefore, the received signal in each channel of the CPDOFDM system can be represented as follows:

$$
\begin{aligned}
& r_{R}(t)=h_{11} s_{R}(t)+\Gamma_{c} h_{21} s_{L}(t)+N_{R}(t) \\
& r_{L}(t)=h_{22} s_{L}(t)+\Gamma_{c} h_{12} s_{R}(t)+N_{L}(t)
\end{aligned}
$$

Where, $N(t)$ means the AWGN and $\Gamma_{c}$ is a reciprocal value of the circular polarization discrimination and it can be written as follow:

$$
\Gamma_{c}=20 \log _{10}\left|\frac{E_{R L}}{E_{R R}}\right|=20 \log _{10}\left|\frac{E_{L R}}{E_{L L}}\right|
$$

In this equation (9), $E_{R R}$ means the strength of the electric fields which is transmitted in the RHCP channel and received by the RHCP channel and so on.

\section{Iv. Characteristics of Conventional OFDM System}

\section{A. Time Delay Effect in Conventional OFDM System}

The time delay of channel occurs interference and loss of orthogonality through the performance attenuation. Figure 4 is BER performance of OFDM system compared to SNR according to time delay of reflection wave [11]. It shows that BER performance more degrades when the delay time of reflection wave becomes more longer. Therefore, guard interval is extended to reduce the effect by the time delay of the reflection wave. However, guard interval extension caused the degradation of transmission efficiency because of undesirable data stream interval. For this reason, it can be convinced that the proposed CPD-OFDM using cross circular polarization wave improved bandwidth efficiency and transmission efficiency.

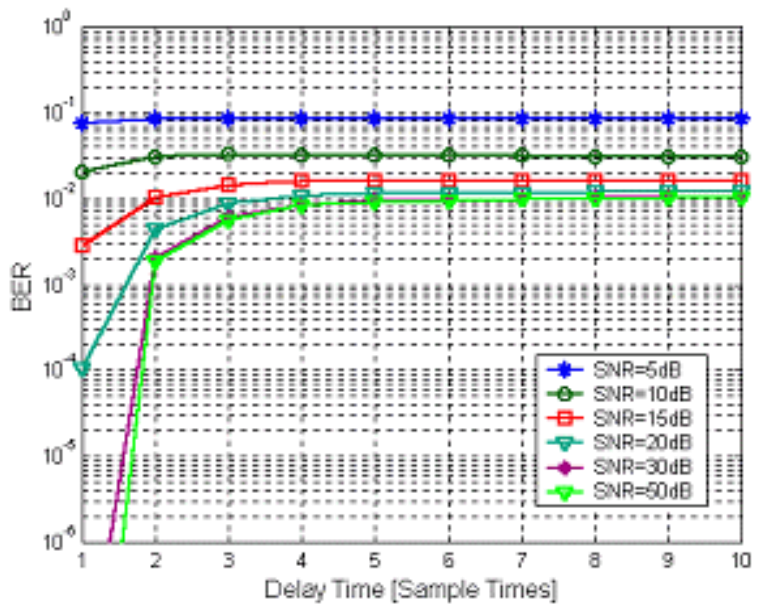

Figure 4. BER performance of the OFDM due to the delay time of the reflected wave $\mathrm{e}^{[11]}$

\section{B. Time Delay Spread}

If impulse $\delta(t)$ is transmitted at time $t=0$, received signal $r(t)$ can be shown as follow:

$$
r(t)=\sum_{i=1}^{n} A_{i} \delta\left(t-T_{i}\right)
$$

$n$ is the number of streams of the signals, $A_{i}$ is the magnitude of the received impulse at the $i^{\text {th }}$ stream and $T_{i}$ is the time delay of the $i^{\text {th }}$ stream. In this case, the impulse response of delay time $T$ shows its characteristic by probability density function $p(T)$, and delay variance $\sigma_{T}$.

$$
\begin{aligned}
& p(T)=\frac{1}{\bar{T}} \exp (-T / \bar{T}) \\
& \sigma_{T}=\sqrt{E\left[T^{2}\right]-E^{2}[T]}
\end{aligned}
$$

In the equation (11), $\bar{T}=E[T]=\int_{0}^{\infty} T p(T) d T$. These equations (11), (12) show that time delay spread $\sigma_{T}$ is reduced by impulse receive delay time $T$ or probability density function is decreased by $p(T)$. Therefore, the time delay spread $\sigma_{T}$ for the channel is reduced according to time delay $T$ and probability density function $p(T)$ which can be also reduced by the removing effect of odd-time reflected waves in cross polarization diversity reception. This is because the time delay spread can be minimized by removing the odd-time reflection waves in circularly polarized wave.

Time delay spread is about $20 \mathrm{~ns}$ on $4 \sim 6 \mathrm{GHz}$ in the indoor channel. But, in circularly polarized wave, time delay spread can be much less than in vertical or horizon polarization by 4.5ns This kind of delay time is much shorter than sampling period of OFDM and it mean that length can be reduced in circularly polarized wave.

\section{System Bandwidth Efficiency}

Transmission rate $R$ and bandwidth $W$ of OFDM system with M-PSK (Multi-Phase Shift Keying) modulation scheme can be represented as follows:

$$
\begin{aligned}
& R=\log _{2} M \times 1 / N T_{s} \times N \\
& W=f_{N-1}-f_{0}+2 \sigma=(N-1) / N T_{s}+2 \delta
\end{aligned}
$$

Therefore, the bandwidth efficiency $\eta$ is as follow [6]:

$$
\eta=\frac{R}{W}=\frac{\log _{2} M}{(1-1 / N) T_{s}+2 \delta T_{s}+G I}
$$

In this equation (14), $\delta$ represents single Bandwidth of subcarrier, it is $\delta=(1+\alpha) / 2 N T_{s}$. The $\alpha$ represents roll-off rate and GI is the bandwidth of guard interval. It can be seen 
that substantial efficient bandwidth is increased by reducing length of the guard interval from the above equation. Usually, the length of guard interval can be up to about $25 \%$ in whole frame. It will be removed after frame synchronization. Thus the bandwidth efficiency can be increased if the length of guard interval is reduced by cross polarization diversity.

\section{v. Simulation Results}

The computer simulation was conducted with the parameters of the IEEE 802.11a [12] by Monte Carlo simulation. The size of the FFT and IFFT are 64 points and sampling rates are $50 \mathrm{~ns}$. The total bandwidth is $20 \mathrm{MHz}$ and the mapping method is QPSK. The convolutional coding with the constraint length of 7 and code rate of $1 / 2$ was adopted. Therefore, the data rate is $12 \mathrm{Mbps}$. Multipath channel with 6rays including direct wave was considered. All 5 reflected waves are considered which have one sampling time interval each other. The reflection loss is considered as $3[\mathrm{~dB}]$ at each reflection.

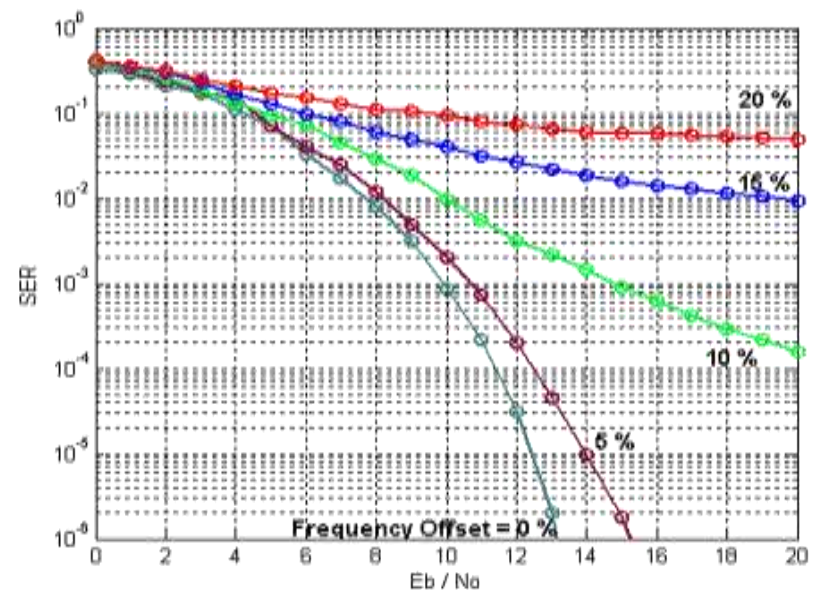

Figure5. SER performance due to frequency offset

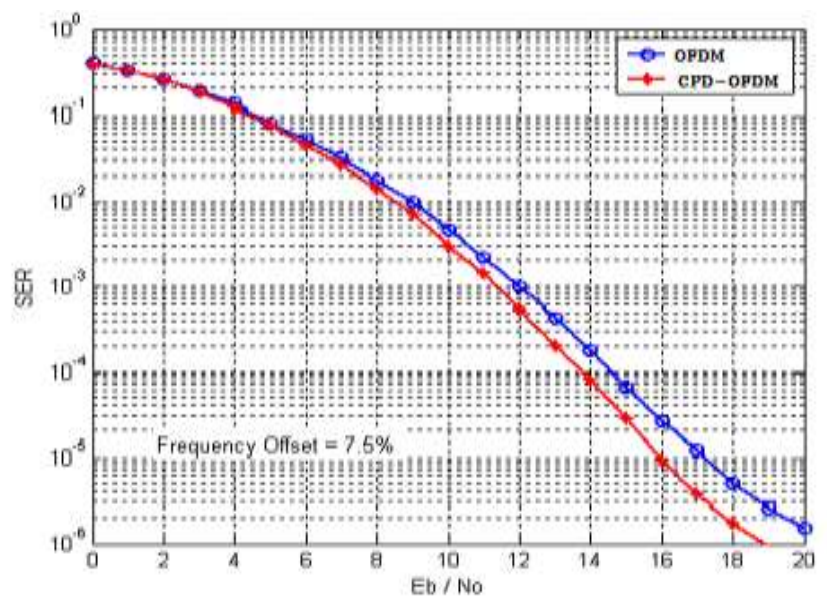

Figure 6. SER Performance when frequency offset is $7.5 \%$
When the frequency offset is presented, both the system performance OFDM and CPD-OFDM with cross polarization diversity structure are estimated. Figure 5 shows the SER (Symbol Error Rate) performance results of the OFDM due to the frequency offsets. From the figure 5, it can be seen that the system performance degrade remarkably according to increasing the frequency offset. In the case of exceeding the 10 percent frequency offset it shows error floor. Figure 6 shows the comparison of the SER performance when frequency offsets happen. From this figure, it can be seen that the performance of the CPD-OFDM system is better than the performance of the conventional OFDM system, and it is more tolerable to the frequency offset than the conventional system.

\section{vI. Conclusions}

In this paper, a modified OFDM system is proposed in order to improve the system performance. The proposed CPDOFDM system has the structure of dividing sub-channels into a RHCP and a LHCP channel. Therefore the orthogonality between sub-channels can be improved by eliminating the overlap of subcarriers using by cross polarization diversity structure. Because of the time delay spread can be minimized and the bandwidth efficiency can be also improved by this cross polarization diversity structure, the proposed CPDOFDM system shows that the power of the ICI caused by the frequency offset can be reduced. It was also found that error floor occurs when frequency offset occur over $10 \%$ in OFDM system. As a result, the proposed CPD-OFDM system minimizes time delay spread and then, the SER performance is improved by about $1 \sim 3[\mathrm{~dB}]$.

\section{Acknowledgment}

This work supported by a Research Grant PuKyoung National University (2017 Years)

\section{References}

[1] Richard Van Nee, Ramjee Prasad, "OFDM for Wireless Multimedia Communication", Arthech House Publishers, pp.229-253, and pp.15-24, 2000.

[2] R.W. Chang, "Synthesis of Band Limited Orthogonal Signals for Multichannel Data Transmission", Bell Syst. Tech. J., Vol.45, pp.17751796, Dec. 1966.

[3] William Y. Zou, Yiyan Wu, "COFDM: AN OVERVIEW", IEEE Trans. on Broadcasting, Vol.41, No.1, pp. 1-8, Mar. 1995.

[4] Rorie O'Neill and Luis B. Lopes, "A Study of Novel OFDM Transmission Schemes for Use in Indoor Environments", IEEE Trans. Com., Vol. E76-B, No.2, pp.909-913, May 1996.

[5] Thierry Pollet, Mark Van Bladel and Marc Moeeclaey, "BER Sensitivity of OFDM Systems to Carrier Frequency Offset and Wiener Phanse Noise", IEEE Trans. on Comm. Vol.43, No.2/3/4, pp.191-193, Feb.-Apr. 1995. 
Proc. of the Fifth International Conference on Advances in Computing, Electronics and Communication - ACEC 2017. Copyright (C) Institute of Research Engineers and Doctors. All rights reserved.

ISBN: 978-1-63248-121-4 doi: 10.15224/ 978-1-63248-121-4-05

[6] L.J. Cimini, "Analysis and simulation of a digital mobile channel using orthogonal frequency-division multiplexing", IEEE Trans. on Comm. Vol.33, No.7, pp. 665-675, July 1985

[7] Theodore S. Rappaport and Dwayne A. Hawbaker, "Wide-Band Microwave Propagation Parameters Using Circular and Linear Polarized Antennas for Indoor Wireless Channels”, IEEE Trans. On Com. Vo.42, No.2, pp.240-245, Feb.1992

[8] Akihiro Kajiwara "On a Circular Polarization Wave Transmission in LOS Indoor Radio Channels" PIMRC A2.6, pp156-159, 1994.

[9] H.C. Jung, S.K. Park, and Deock-Ho Ha, "A Study on Polarization Diversity for the Next Generation Mobile Radio Communications", Journal of Electrical Engineering and Information Science", Vol.3, No.1, pp.117-125, Feb. 1998.

[10] Y.H. Ko, B.O. Kim, Deock-Ho Ha, "Performance of a Novel XCPOFDM system", Proceedings of IEEE $56^{\text {th }}$ VTC 2002 Fall, Vol.1, pp.3841, Sept. 2002.

[11] Deock-Ho Ha, B.O.Kim, "The performance Analysis of A Novel XCPOFDM System Using Cross-Handed Cicular Polarization", Journal of Telecommunications Review, SK Telecomm in Korea, Vol.13, No.3, pp.439-451, Jun. 2003.

[12] IEEE Std802.11a-1999, "Wireless LAN Medium Access Control (MAC) and Physical Layer (PHY) specifications: High-speed Physical Layer in the 5Ghz Band”, IEEE-SA Standard Board, pp. 3-45, Sep. 1999.

About Author (s): Deock-Ho Ha

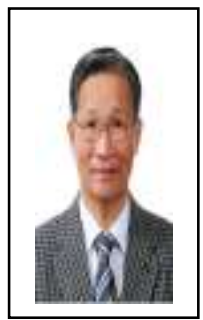

Deock-Ho $\mathrm{Ha}$ received the B.S.E.E. degree, Dept. of Electronics, from Hanyang University in Seoul, Korea in 1979, and received the M.S. and Ph.D. degrees, Dept. of Electronics, from Kyoto University in Kyoto, Japan in 1984 and 1987, respectively. He was a Research engineer with The Central Research Institute of LG Group in Seoul, Korea from 1979 to 1981 , and with The Wireless Research Laboratory of Matsushita Electric Industrial Co. Ltd., in Osaka, Japan from March to August 1987. He was a visiting scholar with the Digicom Laboratory in University of California at Davis from Aug. 1990 to Aug. 1991, and with the Colorado State University at Fort Collins, Colorado USA from Feb. 2000 to Feb. 2001. In Sept. 1987, he joined the faculty of PuKyoung National University in Busan, Korea, where he is a Professor. His research interests include diversity system, modulation technique, indoor/outdoor propagation modeling for the mobile radio communication and indoor wireless LAN.
About Author (s): Kyu-il Han

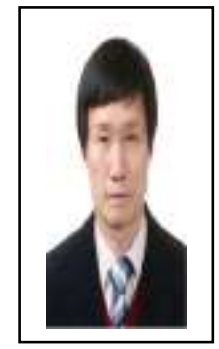

Kyu-il Han received the B.S.M.E. degree from Seoul National University in 1977. He obtained M.S. degree from Ohio State University in 1985 and got Ph.D. degree from Colorado State University in 1989. $\mathrm{He}$ worked as a faculty member since then. And now he is a professer in the Department of Mechanical System Engineering of PuKyuong National University. His research interests are energy efficiency, heat transfer function, and transfer system performance analysis to increase in mechanical system devices. 